\title{
La Declaración de las Naciones Unidas sobre los Derechos de los pueblos indígenas: antecedentes, consecuencias y perspectivas
}

\author{
The United Nations Declaration on the rights \\ of indigenous peoples: background, consequences \\ and prospects
}

\section{Soledad Torrecuadrada García-Lozano*}

\section{Resumen}

Los pueblos indígenas han sido los grandes olvidados en la construcción de las actuales sociedades estatales. En los últimos veinte años, las Naciones Unidas, tras advertir la vulnerabilidad que les caracteriza ha creado diferentes órganos encargados del estudio de la problemática que los rodea, interesándose otros de sus órganos también en ellos. Así, la Asamblea General proclamó en diciembre de 1993 el Primer Decenio Internacional de los Pueblos Indígenas del Mundo, a partir del 10 de diciembre de 1994, comenzando el segundo de los Decenios en enero de 2005 .

Uno de los objetivos perseguidos tanto por las Naciones Unidas como por la OEA es la definición de los derechos de los que son titulares los pueblos indígenas, adoptándose en 2007 la Declaración elaborada en el seno de la organización universal. Este texto no recibió, sin embargo, el

Profesora Titular de Derecho Internacional y Relaciones Internacionales en la Universidad Autónoma de Madrid. <s.torrecuadrada@uam.es>.

Recibido el 20 de agosto de 2009; aceptado el 11 de enero de 2010. 
apoyo que pretendía, y fue objeto de numerosas abstenciones y escasos, pero muy relevantes, votos en contra. El estudio que sigue profundiza en los motivos de esas negativas, así como en sus consecuencias jurídicas.

Palabras Clave: pueblos indígenas, Naciones Unidas, resolución, Declaración, derechos humanos, oponibilidad, tratados.

\section{Abstract}

Indigenous peoples have been largely ignored in the construction of societies organized around states. Over the last twenty years, taking into account their vulnerability, the United Nations has established several bodies charged with studying problems faced by these peoples, and others have also stated relevant interests. In this context, in 1993 the General Assembly proclaimed the First International Decade of the World's Indigenous Peoples, which began on 10 December, 1994, and a second Decade stated in January 2005.

One of the goals of both the United Nations and the OAS is defining the rights of indigenous peoples. The Declaration of such rights, in the frame of the universal organization, was adopted in 2007. However, the text was not as widely supported as expected, and there were many abstentions and also a few but crucial votes against adoption. The following study explores the reasons of such votes and their legal consequences

KEY WorDs: indigenous peoples, United Nations, resolution, Declaration, Human Rights, oponibility, treaties. 


\section{INTRODUCCIÓN}

El objetivo de gran parte de los trabajos realizados sobre los derechos de los pueblos indígenas en el ámbito universal en los últimos quince años fue la adopción de la Declaración sobre los derechos de estos grupos humanos por la Asamblea General de las Naciones Unidas (en adelante, AGNU). El 13 de septiembre de 2007, se producía la votación de un texto en el que, después de más de quinientos años mirando hacia otro lado, se proclaman con carácter general los derechos de estos grupos humanos secularmente olvidados ${ }^{1}$.

El propósito que abanderó los dos Decenios de las Naciones Unidas sobre los pueblos indígenas se lograba así en 2007, marcando un hito que el Foro Permanente de las Naciones Unidas para las Cuestiones Indígenas consideró «uno de los logros más históricos del Segundo Decenio ${ }^{2}$, lo que no obsta para que, a tres años de su inicio, el segundo de ellos agotara su mandato ${ }^{3}$. Sin

1 Es la Resolución 61/295 de la AGNU, de 10 de diciembre de 2007, que contiene la Declaración de las Naciones Unidas sobre los Derechos de los pueblos indígenas.

2 Vid. el Análisis preparado por la secretaría del Foro Permanente de las Naciones Unidas para las cuestiones indígenas: Segundo Decenio Internacional de los Pueblos indígenas del mundo, doc. E/C.19/2009/9, de 4 de marzo de 2009, en p. 5, parágrafo 12 .

3 La resolución 59/174 de la AGNU, de 20 de diciembre de 2004, proclamaba que el Segundo Decenio Internacional de los Pueblos Indígenas del Mundo se iniciaría el 1 de enero de 2005. embargo, aún restan muchas metas por lograr, como lo muestra el hecho de que el objetivo global del Segundo Decenio es, de acuerdo con la Resolución de la AGNU que lo proclama, "continuar fortaleciendo la cooperación internacional para la solución de los problemas a que se enfrentan los pueblos indígenas en esferas tales como la cultura, la educación, la salud, los derechos humanos, el medio ambiente y el desarrollo social y económico, por medio de programas orientados a la acción y proyectos específicos, una mayor asistencia técnica y las actividades normativas pertinentes».

A lo largo de los años transcurridos desde el inicio del primer Decenio se ha producido un importante logro: la sensibilización hacia los pueblos indígenas. Esta sensibilización se ha materializado en las Naciones Unidas (en adelante, NU), donde se ha creado un elevado número de órganos dedicados trabajar monográficamente sobre sus derechos ${ }^{4}$,

4 El ejemplo de NU es ilustrativo, ya que encontramos múltiples foros encargados de este objeto: el Grupo de Trabajo sobre las poblaciones indígenas (que ha elaborado el Proyecto de Declaración de los Derechos de los pueblos indígenas) y el Grupo de Trabajo abierto sobre el Proyecto de Declaración recién citado, ambos incardinados en la estructura de la Comisión de Derechos Humanos. Además, en 2000 se creó el Foro permanente para los pueblos indígenas, órgano asesor del Consejo Económico y Social, del que depende; en 2001 la Comisión de Derechos Humanos nombró a R. Stavenhagen (desde 2008 sustituido por J. Anaya) Relator Especial sobre la situación de los derechos humanos y las libertades fundamentales de los indígenas, con el objeto de estudiar la 
así como en la perspectiva regional ilustrado por la OEA, en cuyo seno se ha discutido mucho sobre los derechos de los pueblos indígenas y se ha avanzado considerablemente en su definición ${ }^{5}$. La adopción de la Declaración americana se está demorando sobre el calendario previsto inicialmente debido a las dificultades en la búsqueda de consensos entre las perspectivas indígena y es-

discriminación contra las mujeres indígenas, así como las violaciones de los derechos humanos y las libertades fundamentales de que son víctimas los niños indígenas. A todo los anterior hay que sumar la elaboración de diversos informes relacionados con los pueblos indígenas sobre temas tan variados como la protección del patrimonio de los pueblos indígenas, la relación de los pueblos indígenas con su territorio, tratados, acuerdos y otros arreglos entre los Estados y las poblaciones indígenas... Además, no podemos olvidar que en la estructura de NU hay otros foros que también se ocupan aunque sea sectorialmente de los pueblos indígenas, es el caso del PNUD, la FAO, el UNICEF.

5 Los trabajos para lograr una Declaración en este sentido comenzaron en 1989 cuando la Asamblea General de la Organización solicitó a la Comisión Interamericana de Derechos Humanos la preparación de un Proyecto que la Comisión concluyó en 1997 (Vid. en OEA/Ser.G, Consejo Permanente/doc.2878/97 corr.1, de 1 abril 1997). La Asamblea de la OEA entendió que debían incorporarse las observaciones de los Estados miembros, así como las del Comité Jurídico Interamericano y del Instituto Indigenista Interamericano. El Texto Consolidado del Proyecto de Declaración preparado por la Presidencia del Grupo de Trabajo es de 17 de junio de 2003 (vid. OEA/Ser.K/XVI. Doc. GT/ DADIN/doc.139/03, de 17 de junio de 2003). tatal (entre el 30 de noviembre y el 2 de diciembre se celebró la duodécima reunión con este propósito). Como se explicación más adelante quizá uno de los rechazos más frontalmente expuestos al contenido de la Declaración americana se materializa en la expresión de la opinión de Estados Unidos en la Décima reunión de negociación para la búsqueda de consensos ${ }^{6}$.

La sensibilización hacia los problemas de los pueblos indígenas se ilustra igualmente en publicaciones científicas, en las noticias que nos transmiten los medios de comunicación, que ahora se hacen eco de los acontecimientos que afectan a la vida de los grupos indígenas y de las difíciles condiciones en que sobreviven. Una muestra más del interés generalizado que han despertado los problemas indígenas es la aparición, en países que como España carecen de población indígena ${ }^{7}$, de organizaciones, centros o asociaciones que persiguen facilitar alguna modalidad de ayuda a

6 Vid. especialmente el Doc. GT/DADIN/ doc. 301/07.

7 Por señalar solo dos ejemplos, recordaré la inauguración, en diciembre de 2000, del Centro Internacional de Información y Documentación de los pueblos indígenas, gracias al apoyo del Ayuntamiento de Vitoria-Gasteiz y con la colaboración de la Diputación Foral de Álava, creado con el propósito de mantener y sistematizar un fondo bibliográfico y documental sobre temas indígenas. Antes ya existía la Fundación de artistas e intelectuales por los pueblos indígenas de Iberoamérica, que articula mecanismos de apoyo a las iniciativas de desarrollo que las mismas comunidades indígenas propongan y elaboren. 
estos pueblos o en un ámbito general, el número de foros internacionales que se encuentran trabajando sobre esta cuestión ${ }^{8}$.

Sin embargo, mucho antes de producirse la visibilidad de los pueblos indígenas hubo una organización internacional que ha intentado promover y proteger sus derechos: la OIT. A principios de los años veinte del siglo XX, mientras la Sociedad de Naciones cerraba sus puertas a algunos de sus líderes cuando pretendieron transmitirle la difícil situación en que vivían los grupos a los que representaban ${ }^{9}$, esta organización de naturaleza sectorial se interesaba por ellos. En su seno se han celebrado dos modalidades de convenios: (1) los que definen los derechos exclusivamente laborales de los trabajadores indígenas -por tanto, in-

$8 \quad$ España ha ratificado el Convenio 169 de la OIT el 15 de febrero de 2007 y la Agencia Española de Cooperación Internacional (AECI) desarrolla un interesante trabajo a estos efectos.

$9 \quad$ En 1923, el Jefe cayuga Deskahen, visitó la sede de la Sociedad de Naciones (SdN) como representante de las Seis Naciones de Iroqueses, sin que su desplazamiento tuviera resultados prácticos. Al año siguiente, 1924, W.T. Ratana, dirigente religioso maorí viajó hasta Londres con la intención de pedir ayuda al Rey Jorge ante el incumplimiento del Tratado de Waitangi. Como no se le recibió, su delegación fue a Ginebra, recibiendo de la SdN un trato muy parecido al cosechado en Londres. Un año más tarde, en 1925, Ratana acudió personalmente a Ginebra, aunque tampoco fue recibido por esta organización. Vid. http://www.un.org/spanish/indigenas/2003. dividuales-, realizados en un momento inicial; y, (2) más recientemente, y una vez que los derechos humanos exceden a la jurisdicción interna, los derechos (no solo laborales) de las denominadas poblaciones indígenas -derechos colectivos-.

En tanto que precedente de la Declaración de las Naciones Unidas los Convenios que nos interesan son los que pertenecen a la segunda categoría apuntada $^{10}$ : los Convenios 107 y 169. El primero de ellos que se relaciona con la protección e integración de las poblaciones indígenas y de otras poblaciones tribales y semitribales en países independientes ${ }^{11}$, define junto

10 Dentro de la primera, los convenios concluidos sobre diferentes aspectos laborales, encontramos el que proscribe el reclutamiento de trabajadores indígenas y otros que regulan los contratos de trabajo de los trabajadores indígenas; pretenden impedir las sanciones penales impuestas a los trabajadores indígenas; limitan la duración máxima de los contratos de trabajo de los trabajadores indígenas; o un nuevo convenio que revisa y complementa el anteriormente concluido sobre abolición de las sanciones penales por incumplimiento de contrato de trabajo por parte de trabajadores indígenas, reduciendo el margen de discrecionalidad estatal permitido en aquel. Aunque en la actualidad estos tratados internacionales no estén vigentes, son indicativos de los abusos de los que eran víctimas en la primera mitad del siglo $\mathrm{XX}$ los trabajadores indígenas.

11 Es el Convenio 107, aprobado el 26 de junio de 1957, en vigor desde el 2 de junio de 1959. Hasta el 16 de julio de 1986, fecha en la que recibió la última ratificación, habían manifestado el consentimiento en vincularse por él los siguientes Esta- 
con los derechos de carácter laboral, otros de los que son titulares esos grupos humanos y que exceden el ámbito material apuntado (como los derechos sobre las tierras, la formación profesional, la protección de artesanía y de las industrias rurales, la seguridad social y la salud o la educación y los medios de información). En todo caso, debido al momento de su celebración, el objetivo último que perseguía este tratado, tal y como se desprende de su mismo título, era la asimilación de los indígenas a los sectores sociales mayoritarios ${ }^{12}$.

Años más tarde, fruto de la revisión del Convenio 107 se logró un nuevo texto sobre pueblos indígenas y tribales en países independientes $\left(\mathrm{el} \mathrm{n}^{\circ} 169\right)^{13}$,

dos: Angola, Argentina, Bangladesh, Bélgica, Brasil, Colombia, Costa Rica, Cuba, Ecuador, Egipto, El Salvador, Ghana, Guinea-Bissau, Haití, India, Iraq, Malawi, México, Pakistán, Panamá, Paraguay, Perú, Portugal, República Dominicana, República Arabe Siria y Túnez.

12 Si tenemos en cuenta los propósitos y fines del indigenismo oficial de la época, observaremos que el objeto del Convenio de la OIT no se apartaba en gran medida de ellos. De acuerdo con A. Caso, aquel pretendía la aceleración y consolidación de la integración de la población indígena en el Estado.

En el que estaban ubicados, además de la promoción del desarrollo económico y social de las comunidades indígenas. Vid. Caso A., (1971) La Comunidad indígena, México, Sepsetentas Diana p. 139.

13 El Convenio 169 se aprobó el 27 de junio de 1989, y su texto está en vigor desde el 5 de septiembre de 1991. A 23 de diciembre de 2009 son partes en él veinte Estados: Argentina, Bolivia, Brasil, Chile, Colombia, Costa Rica, Dinamarca, Domi- que toma como punto de partida -reflejo de la nueva percepción- el respeto de la diversidad cultural para definir los derechos de que son titulares los pueblos indígenas. Es un tratado internacional que enuncia los derechos básicos de estos grupos humanos e incorpora obligaciones de resultado dirigidas a los Estados partes -la adopción de medidas concretas para lograr las finalidades propuestas-, aunque en algunas ocasiones introduce cierto margen de discrecionalidad estatal ${ }^{14}$.

En la actualidad, ambos Convenios están vigentes, ya que son tratados sucesivos sobre la misma materia ${ }^{15}$. Sin

nica, Ecuador, España, Fiji, Guatemala, Honduras, México, Nepal, Noruega, Holanda, Paraguay, Perú y la República Bolivariana de Venezuela.

14 Esto ocurre, por ejemplo en relación con la enseñanza infantil de la lectura y la escritura en la propia lengua de los afectados (art. 28.1). En este caso, «siempre que sea viable, deberá enseñarse...» en los idiomas referidos; "cuando ello no sea viable» la única obligación que se impone al Estado parte es la consulta con los pueblos interesados para procurar la «adopción de medidas que permitan alcanzar este objetivo». Hemos de tener en cuenta que quien califica la viabilidad o no de este tipo de enseñanza es el propio Estado afectado, por tanto, lo hará discrecionalmente y atendiendo a razones múltiples, entre ellas por ejemplo, la política económica o las prioridades presupuestarias.

15 El primero en celebrarse en el tiempo (el Convenio 107) incorpora una cláusula reguladora de la relación entre ellos, que establece que la adquisición del estatuto de parte en el Convenio revisor supondrá la denuncia inmediata de aquel (art. 36), manteniéndose en vigor para aquellos Estados que, siendo partes en el primero, 
embargo, a pesar de los avances que supusieron estos Convenios, de ellos derivan dos problemas: 1) la ausencia de mecanismos específicos de control hace que puedan beneficiarse únicamente de lo establecido con carácter general en la Constitución de la OIT; 2) el relativismo propio del derecho internacional. En relación con el primero de ellos, si bien no es posible la presentación de quejas por parte de individuos u organizaciones no gubernamentales, sí se prevé en el mismo texto constitucional (art. 24) que empleadores, organizaciones nacionales o internacionales, $\mathrm{o}$ incluso particulares denuncien a un Estado que no haya observado o garantizado satisfactoriamente la aplicación de un convenio en el que es parte. Los resultados, sin embargo, pueden ser escasos al limitarse a la publicidad de la demanda recibida y, en su caso, de la respuesta remitida por el Estado presuntamente infractor $(\text { art. } 25)^{16}$.

no hayan ratificado el segundo. Por otra parte, evidentemente, los Convenios de la OIT contienen estándares mínimos de protección, que los Estados partes, a través de sus respectivas legislaciones internas, pueden superar. Este incremento se detecta ya no solo en los sistemas normativos nacionales de los Estados que cuentan con el estatuto de partes en estos textos convencionales, sino también en otros que no han manifestado su consentimiento en obligarse por ellos. En este sentido, recordemos que, por ejemplo, en Nueva Zelandia -que no es parte en el Convenio $\mathrm{n}^{\circ}$ 169-, las madres maoríes han conseguido que se garantice por Ley la financiación de la educación indígena.

16 El art. 25 de la Constitución de la OIT indica que la reclamación individual for-
La dificultad apuntada en segundo lugar resulta más difícil de resolver. En efecto, el relativismo del derecho internacional convencional solo se supera con su consolidación como norma general, ya sea por el incremento (más que multiplicación) del número de sujetos obligados por él; o ya porque los contenidos en él incorporados se convierten en norma consuetudinaria. Con la Declaración de NU se pretendía establecer el elemento espiritual de una costumbre general que, unido a la práctica estatal, terminara consolidando su contenido como norma consuetudinaria general, alejándonos así de la relatividad propia de los tratados internacionales. En la actualidad, las laxas obligaciones in-

mulada se comunica al Consejo de Administración de la Organización, la que tiene la facultad de transmitírsela al Estado miembro frente al que se presenta aquella, a los efectos de que declare lo que considere oportuno respecto del contenido de la reclamación individual formulada. Si en un plazo prudencial no responde o su respuesta no se considera satisfactoria, el Consejo de Administración puede hacer pública la reclamación y la respuesta si se hubiere recibido. En otros supuestos, el Consejo puede decidir acerca del fondo de la reclamación, correspondiendo a la Comisión de expertos en aplicación de Convenios y Recomendaciones el seguimiento de las medidas tomadas por el gobierno para dar cumplimiento a aquella decisión. En todo caso, la Comisión de cuestiones jurídicas y normas internacionales del trabajo informa en su $288^{a}$ reunión, de noviembre 2003 (parágrafos 20 y ss.) que los arts. 24 y 25 de la Constitución son susceptibles de mejoras y se han formulado sugerencias al respecto. Vid. Doc. GB.288/LILS/1 pp. 7-ss. 
corporadas en los Convenios de la OIT resultan exclusivamente exigibles a los Estados partes, que no son demasiados. De todos modos, con independencia de cuales sean las deficiencias de los Convenios de la OIT o de las críticas que puedan merecer por variados motivos, lo cierto es que, de momento, los únicos textos internacionales en vigor o que en algún momento han tenido vigencia, son los celebrados bajo sus auspicios. Reconozcámosle por tanto todo el mérito que deriva de esta consideración.

En otro orden de consideraciones, uno de los problemas con que nos encontramos cuando estudiamos los derechos de los pueblos indígenas (superada la inexistencia de una definición generalmente aceptada ${ }^{17}$ ), es la heterogeneidad de la realidad que identifica, debido a su pluralidad. En el mundo habitan aproximadamente 370 millones de personas de origen indígena, distribuidas en 5.000 culturas (de las aproximadamente 6.000 que hay en el mundo). Tienen presencia en todos los continentes, incluso en Europa, donde debemos considerar que aproximadamente unos 80.000 indígenas sami viven en las regiones árticas de Noruega, Suecia, Finlandia y la península rusa de $\mathrm{Kola}^{18}$. A ellos hay

17 Vid. sobre la definición de pueblo indígena, Torrecuadrada S., Los pueblos indígenas en el orden internacional, Madrid, Dyckinson, 2001.

18 Cerca de la mitad de los indígenas europeos habitan en países miembros de la Unión Europea, sin que este grupo se caracterice por unas condiciones de vida más que añadir los casi 60.000 indígenas inuit de Groenlandia ${ }^{19}$ que en noviembre de 2008 votaron mayoritariamente (un $75.54 \%$ ) en favor de ampliar el estatuto de autonomía, que les concede el derecho de autodeterminación de Dinamarca $^{20}$.

Por otra parte, pueblos indígenas son tanto los no contactados o en situación de aislamiento ${ }^{21}$ como aquellos que tienen relevancia y participación políti$\mathrm{Ca}^{22}$; los que son numéricamente mino-

favorables que las de quienes habitan otras regiones geográficas del mundo.

19 El 25 de mayo de 2004 los inuit de Thulé depositaron una demanda contra Dinamarca ante el Tribunal Europeo de Derechos Humanos (18584/04), por la que reivindicaban la devolución de las tierras de las que fueron expulsados en 1953. La demanda fue desestimada en la Sentencia de 12 de enero de 2006, por incompetencia del Tribunal para conocer de unos hechos producidos antes de que Dinamarca adquiriera la condición de parte en la Convención (3 de septiembre de 1953) y en el Protocolo 1 (18 de mayo de 1954). En todo caso, el Tribunal apreció el interés danés por resolver, mediante una compensación económica, la reubicación de la tribu, aunque las partes no lograron un acuerdo acerca de la cuantía de la misma.

20 Ver en este sentido la noticia difundida por las agencias de prensa el 26 de noviembre de 2008, vid. http://www.elpais. com.

21 De acuerdo con S. Manchineri, Coordinador General de la Coordinadora de las Organizaciones Indígenas de la Cuenca del Amazonas (COICA) «alrededor de 64 pueblos indígenas viven en situación de aislamiento en países como Ecuador, Brasil y Perú», vid. en http://www.coica.org . Vid. infra epígrafe V.

22 En América Latina, además del caso emblemático de Bolivia y su Presidente, Evo 
ritarios en el territorio estatal como los que son amplia mayoría. Entre ambos extremos existe una gran variedad de realidades que no resultan comparables entre sí. No se enfrentan a los mismos problemas los indígenas de Estados Unidos que los que se encuentran en aislamiento voluntario por ejemplo en las selvas brasileñas y dentro de un mismo Estado las condiciones en que habitan son dispares, no es lo mismo la tribu de los semínola en Florida Estados Unidos, que la de los navajo (que también tienen alguna participación en el juego) o los inuit de Alaska.

A pesar de la diversidad característica de los pueblos indígenas, su regulación internacional es única. Con ella se intenta establecer un sistema de garantías mínimas de protección para estos grupos en situación de especial vulnerabilidad. En todo caso, no podemos olvidar que los pueblos indígenas están integrados por personas que son

Morales (aymara), hay distintos Estados como, entre otros Ecuador, cuyos gobernantes han recibido un importante apoyo indígena. Un año después de que el coronel Lucio Gutiérrez asumiera la Presidencia ecuatoriana, en enero de 2003, el desencanto indígenafue el inicio del fin de su mandato. No olvidemos que J. Mahuad, fue derrocado en 2000 por una revuelta civil-militar, que contó con una decisiva participación indígena. Sobre el movimiento indígena en Perú, vid. (2002) Dávalos, Pablo «Movimiento indígena ecuatoriano: Construcción política y epistémica", en: D. Mato (coord.): Estudios y Otras Prácticas Intelectuales Latinoamericanas en Cultura y Poder, Caracas, Universidad Central de Venezuela, 2002. titulares de los derechos humanos y las libertades fundamentales en la misma medida que todos los demás ${ }^{23}$.

En las páginas que siguen nos vamos a centrar en el estudio de la Declaración de las Naciones Unidas aprobada en 2007, muy especialmente en el resultado de su votación, las causas que motivaron los rechazos (escasos desde una perspectiva cuantitativa, pero cualitativamente muy importantes para los fines que perseguimos) y sus consecuencias.

\section{POR FIN CONTAMOS CON UN INSTRUMENTO UNIVERSAL SOBRE LOS DERECHOS DE LOS PUEBLOS INDÍGENAS}

Como se indicó supra, los trabajos de elaboración de la Declaración de las Naciones Unidas sobre los derechos de los pueblos indígenas se iniciaron con el propósito de evitar, en la medida de lo posible, el relativismo característico del derecho internacional en general y de los tratados internacionales en forma más concreta. El resultado de estas ta-

23 Señalemos por ejemplo, por este concepto, la Declaración Universal de los Derechos Humanos y los Pactos Internacionales de Derechos Humanos. Además, aquellos que habitan en el continente americano, gozan de la protección proporcionada por el sistema interamericano de derechos humanos, mientras los ubicados en el continente europeo, del Convenio de Roma de 1950 sobre la materia. Por tanto, las personas indígenas gozan de la misma protección jurídica individual, en lo que a derechos humanos se refiere, que los que no seamos considerados tales. 
reas se incorpora en una Resolución de la Asamblea General (carácter recomendatorio), con la confianza de que con el transcurso del tiempo se convirtiera en una norma general (como ocurriera casi sesenta años antes con la Declaración Universal de Derechos Humanos). Sin embargo, el día de la adopción se quebraron nuestras esperanzas, pues para ello precisábamos la ausencia de votos en contra o que, de existir, fueran poco relevantes. Cierto es que las oposiciones no fueron muy numerosas (solo 4 de los 158 votos emitidos), derivando el fiasco no de la cantidad, sino de la identidad de quienes decidieron votar negativamente, al tratarse de Estados (todos ellos fruto de la colonización británica) que cuentan con una importante población indígena: Estados Unidos (que ya anunció en 2008 que no apoyaría la Declaración de la OEA sobre este mismo objeto), Australia, Canadá y Nueva Zelandia.

Ahora nos detendremos a considerar dos aspectos relacionados con ese resultado: por una parte, los motivos que han conducido a los Estados a votar de la forma en que lo hicieron; y, de otra, los inconvenientes que pueden derivar de la votación para la consolidación de la Declaración como derecho internacional general.

\section{A. Los motivos de la oposición a la adopción de la Declaración}

Sorprende que quienes se opusieron a la aprobación de la Declaración fueran precisamente Estados que cuentan con una legislación y jurisprudencia avanzada y progresista en la protección de los derechos de los pueblos indígenas. El motivo de su rechazo se encuentra en los idénticos aspectos, algunos de los cuales son de especial interés para los pueblos indígenas. Se trata del reconocimiento tanto de la libre determinación y su contradicción con la integridad territorial como de los derechos particulares sobre sus territorios, mucho más amplios que los que se reconocen a otros propietarios no indígenas de los suyos (en concreto, a los recursos que se encuentren en las tierras indígenas); el excesivo alcance del que se dota al consentimiento libre e informado de los pueblos indígenas; el desconocimiento de los derechos de terceros derivado de la amplitud de derechos que se conceden a los pueblos indígenas; íntimamente relacionado con el anterior, la primacía del derecho consuetudinario sobre el derecho nacional; y, por último, el desequilibrio (o la asimetría) entre las obligaciones y los derechos de los pueblos indígenas.

El principal motivo argüido por Australia en la explicación de su voto contrario a la Declaración fue el reconocimiento a la libre determinación y su contradicción con la integridad territorial, aludiendo a los presupuestos de aplicación del derecho en cuestión en su más amplia extensión. Ello a pesar de que en el texto votado en septiembre de 2007 no solo se reconoce exclusivamente la vertiente interna de 
este derecho (tal y como expresamente indica la declaración en su artículo $4^{24}$ ), lo que se traduce en la autonomía o autogobierno, sino que además incorpora una cláusula de salvaguardia de la integridad territorial del Estado (art. 46.125), sin la cual los Estados difícilmente hubieran permitido su adopción. De cualquier modo, no olvidemos que nos referimos a pueblos que habitan en el interior de las fronteras de un Estado, a los que resulta aplicable la cuarta fórmula de ejercicio del derecho a la libre determinación incorporada en la resolución 2625 (XXV): la autonomía o el autogobierno, por lo que el alcance del reconocimiento del derecho a la libre determinación desde la perspectiva internacional exclusivamente podía ser el que se ha incorporado. La discrepancia estatal en este sentido no es sustantiva sino formal, puesto que el origen del rechazo no radica en el contenido -au-

24 El artículo 4 de la Declaración contiene la siguiente redacción: «Los pueblos indígenas, en ejercicio de su derecho a la libre determinación, tienen derecho a la autonomía o al autogobierno en las cuestiones relacionadas con sus asuntos internos y locales, así como a disponer de medios para financiar sus funciones autónomas».

25 Artículo 46.1: «Nada de lo contenido en la presente Declaración se interpretará en el sentido de que confiere a un Estado, pueblo, grupo o persona derecho alguno a participar en una actividad o realizar un acto contrarios a la Carta de las Naciones Unidas, ni se entenderá en el sentido de que autoriza o alienta acción alguna encaminada a quebrantar o menoscabar, total o parcialmente, la integridad territorial o la unidad política de Estados soberanos e independientes». togobierno- incorporado en el texto de la Declaración, sino en las posibles interpretaciones que, del principio de la libre determinación, pudieran hacerse. En la confección del texto de la Resolución se decide utilizar el concepto de libre determinación interna por estimar que satisfaría los intereses tanto de los pueblos indígenas (que quisieran verse titulares de ese derecho ${ }^{26}$ ) como de los Estados (con la limitación derivada del adjetivo interno y de la cláusula de

26 En realidad, con carácter general, la reivindicación indígena se limita al establecimiento de un sistema de autogobierno en sus territorios, lo que coincide con lo establecido en la Declaración. El logro del autogobierno lo obtuvieron los esquimales de Groenlandia en 1979 (en virtud del cual decidieron en 1982 abandonar la Comunidad Europea) o por los inuit cuando el 1 de abril de 1999, Nunavut se convirtió oficialmente en un territorio autónomo de Canadá. Groenlandia es la isla del hielo, dado que el $84 \%$ de su superficie lo es. Es la segunda reserva de hielo del planeta. Su economía se basa principalmente en el marisco, la pesca, la artesanía y las pieles. Los thule de quienes descienden directamente los inuit groenlandeses poblaron la isla desde el siglo X. Los escandinavos llegan más tarde. Noruega anexionó oficialmente Groenlandia en el siglo XIII, recibiendo Dinamarca la isla en el siglo XVII. Estuvo controlada por Estados Unidos durante la segunda guerra mundial Vid. supra nota $\mathrm{n}^{\circ} 10$, el referéndum de 2008. Nunavut fue el resultado de más de 30 años de negociaciones entre los inuits del este y del centro del Ártico, el Gobierno de los territorios del Noroeste y el Gobierno de Canadá. Su economía está basada en la pesca y la caza junto con otras como la contribución de la industria minera y petrolera, arte y artesanía y turismo. 
salvaguardia antes indicada). A pesar de ello, esta licencia ha sido el origen del rechazo o la abstención de algunos Estados.

En todo caso, aunque la Declaración proclamara la libre determinación con un alcance externo, en la mayoría de los casos resulta materialmente impracticable, al encontrarnos con supuestos como el de Venezuela que reúne una población indígena de aproximadamente 300.000 personas divididas en 30 etnias diferentes y 1500 comunidades indígenas repartidas por el país ${ }^{27}$. La situación estructural del país parece impedir la posibilidad del ejercicio de este derecho más allá de la autonomía ${ }^{28}$; pero cuan-

27 Al mismo tipo pertenecerían Brasil, Colombia o Estados Unidos, por citar solo algunos ejemplos.

28 Un caso interesante en este punto es el de Hawaii, donde si se escuchan voces que defienden el ejercicio de la libre determinación ahora externa, pero no en razón al origen indígena, sino porque fue anexionada por la fuerza por Estados Unidos al final del siglo XIX. Hawaii fue anexionado a finales del siglo XIX, procedimiento ilícito internacionalmente para la adquisición territorial. Desde hace algunos años, un sector de la población hawaiana (curiosamente el formado por los indígenas hawaianos) reivindica el ejercicio a la libre determinación, por entenderse objeto de dominación por parte del Estado que lo anexionó. Más si cabe después de que el Presidente Clinton expresara públicamente sus excusas por el trato del que fue objeto una nación democrática y moderna como era Hawaii cuando se convirtió en parte de Estados Unidos. Hay una biografía de la última reina de Hawaii en la que se muestran todos los sucesos. También se interpuso una demanda arbitral (ante la do no es este el caso, las solicitudes de autogobierno de los pueblos indígenas difícilmente han recibido una respuesta afirmativa, debido al temor a que fuera el inicio de la desintegración nacional. En otras ocasiones, el Estado carece de capacidad para imponer el derecho general a las comunidades indígenas que se encuentran alejadas, geográfica o políticamente, de los centros de poder, cuando no resultan inexistentes por invisibles para las autoridades (o los intereses) estatales.

Junto con el anterior, otro motivo de oposición a la Declaración se encuentra en la que es la principal reivindicación indígena: el reconocimiento de derechos particulares sobre sus territorios. La Declaración de las Naciones Unidas proclama, por una parte, que «Los pueblos indígenas tienen derecho a las tierras, territorios y recursos que tradicionalmente han poseído, ocupado o utilizado o adquirido", junto con el de "poseer, utilizar, desarrollar y controlar las tierras, territorios y recursos que poseen en razón de la propiedad tradicional u otro tipo tradicional de ocupación o utilización, así como aquellos que hayan adquirido de otra forma»; lo que, en el caso de algunos

Corte Permanente de Arbitraje) para que se determinaran las cuestiones relativas a la soberanía territorial sobre las islas. El Tribunal concluyó su falta de competencia para conocer de la cuestión planteada, motivando su rechazo en que Estados Unidos no era parte en el proceso. El demandante es Lance Paul Larsen, residente en Hawaii c. «El Reino Hawaiano por su Consejo de Regencia» 
Estados, como Nueva Zelandia, supone la práctica totalidad del territorio nacional ${ }^{29}$. Por otra parte, se reconocen los derechos colectivos de los pueblos indígenas no solo sobre las tierras con el mismo alcance que para el resto de los propietarios individuales, sino también sobre los posibles recursos que puedan encontrarse en ellas, lo que contradice a la mayoría de las Constituciones estatales que establecen la propiedad estatal sobre estos recursos.

El reconocimiento de los derechos sobre las tierras y, en concreto el de su titularidad colectiva, es el aspecto que con mayor interés reivindican los pueblos indígenas, por entender que proporcionan beneficios a toda la comunidad ${ }^{30}$. Esta importancia se subraya en el preámbulo de cuantos textos se dirigen a proteger los derechos de estos grupos humanos. Así, el parágrafo quinto del Preámbulo del texto

29 Vid. explicación de voto del representante de Nueva Zelandia en A/61/PV.107

30 Vid. en este sentido por todos, el Estudio del Problema de la discriminación contra poblaciones indígenas, realizado por $\mathrm{R}$. Martínez Cobo -cit. supra- en cuyos parag. 196-197 del vol. V observa «la relación especial profundamente espiritual de los pueblos indígenas con sus tierras como algo básico en su existencia como tales. Para los indígenas, la tierra no es meramente un objeto de posesión y producción. La relación integral de la vida espiritual de los pueblos indígenas con la Madre Tierra, con sus tierras, tiene muchas implicaciones profundas. Además, la tierra no es mercadería que pueda apropiarse, sino elemento material del que debe gozarse libremente». de la Declaración de la OEA (199731), referido al territorio y la supervivencia indígenas, reconoce «que para muchas culturas indígenas sus formas tradicionales colectivas de control y uso de tierras, territorios, recursos, aguas y zonas costeras son condición necesaria para su supervivencia, organización social, desarrollo, bienestar individual y colectivo; y que dichas formas de control y dominio son variadas, idiosincráticas y no necesariamente coincidentes con los sistemas protegidos por las legislaciones comunes de los Estados en que ellos habitan».

La redacción de la Declaración de las Naciones Unidos en este punto responde a las reivindicaciones indígenas centradas principalmente en la recuperación de las tierras de las que fueron desposeídos; el reconocimiento de los derechos -sagrados, básicos y fundamentales a poseer, desarrollar, utilizar, controlar y ocupar sus tierras ${ }^{32}$, , colectivos, tanto sobre ellas como en relación con los recursos tradicionales que puedan encontrarse en este elemento incluso los arqueológicos-; además del reconocimiento como derecho al respe-

31 En el texto consolidado, de 2003, la primera parte de la redacción transcrita se mantiene en el parágrafo cuarto del mismo preámbulo.

32 Es una frase tomada de una declaración del representante del Consejo Internacional de Tratados Indios al Grupo de Trabajo de la Consulta Mundial de las $\mathrm{Na-}$ ciones Unidos sobre el Derecho al Desarrollo (parágrafo 94 del Doc. UN E/ CN.4/2000/84, de 6 de diciembre de 1999). 
to a la integridad y conservación de su hábitat natural -incluyéndose en este punto la protección medioambiental- ${ }^{33}$.

En todo caso, la recuperación del territorio ancestral de los pueblos indígenas es una cuestión de difícil solución, tanto si nos encontramos con una desposesión alejada en el tiempo como si resulta reciente. En el primer caso, porque la devolución puede chocar con la defensa de los presuntos derechos adquiridos con el transcurso del tiempo o, incluso, con el interés general. En el segundo, en la medida en que se produce en un contexto de conflicto abierto entre las partes que, normalmente, es preciso resolver con carácter previo. Sí es cierto que en este punto se va avanzando gracias, en muchos casos, al apoyo de órganos internacionales. En el ámbito latinoamericano, la Corte Interamericana de Derechos Humanos, en la Sentencia de 2001 recaída en el asunto de la Comunidad Mayagna (Sumo) Awas Tingni contra Nicaragua ${ }^{34}$, ha resultado de extraordinaria utilidad. El objeto de la controversia se encuentra en la demora del Gobierno nicaragüense tanto

33 Recordemos que el Comité de Derechos Humanos de Naciones Unidas ha reiterado la íntima relación existente entre el derecho a la tierra y a los recursos que se encuentran en ella y el ejercicio del derecho a la libre determinación de los pueblos, en tanto que los primeros son un elemento necesario para el correcto ejercicio del segundo.

34 Vid. Sentencia de 31 de agosto de 2001, Serie C, $\mathrm{n}^{\circ} 79$, vid. en http://www.corteidh. or.cr. en la demarcación de las tierras de los awa tigni como en su reconocimiento jurídico (previsto en el art. 5 de la Constitución nicaragüense ${ }^{35}$ ). Además de lo anterior otorgó una concesión a una empresa forestal coreana para que explotara casi 65.000 mil hectáreas de tierra ocupadas tradicionalmente por esta comunidad indígena.

La Corte Interamericana en esta Sentencia formula una afirmación de extraordinario interés: todos los Estados que reconozcan constitucionalmente los derechos indígenas sobre sus tierras y no establezcan mecanismos eficaces y reales de delimitación están vulnerando, con esta omisión, no solo su propia Constitución sino también la Convención Americana de Derechos Humanos. De acuerdo con lo anterior, los awas tingni son propietarios de sus tierras (porque así lo dice la Constitución de Nicaragua), vulnerando la ausencia de mecanismos efectivos

35 En concreto se refiere a la comunidad mayagna (Sumo) awas tingni y a la Constitución Nicaragüense cuyo art. 5 reconoce el derecho de los pueblos indígenas a «mantener las formas comunales de propiedad de sus tierras y el goce, uso y disfrute de las mismas, todo de conformidad con la ley». La Corte entiende que de este precepto se desprende el reconocimiento de la propiedad colectiva de las tierras indígenas y la existencia de títulos de propiedad en beneficio de los awas tingni, al establecer que «la posesión de la tierra debería bastar para que las comunidades indígenas que carezcan de un título real sobre la propiedad de la tierra obtengan el reconocimiento oficial de dicha propiedad y el consiguiente registro». 
adecuados para la delimitación, demarcación y titulación de las tierras indígenas, entre otros, el derecho a la propiedad privada de todas las personas sometidas a la jurisdicción de los Estados Partes sin discriminaciones (art. 21 de la Convención). De este modo, se subraya la importancia de la existencia de normas internacionales reguladoras de esta materia, cuya ausencia dejaría en manos del Estado incumplidor la exclusiva competencia para conocer el asunto en el que él mismo es parte interesada, al ser quien otorga la concesión de explotación.

En la actualidad existen movimientos demarcadores de tierras especialmente en América Latina. Es el caso de Canadá, Paraguay ${ }^{36}$, Brasil o la República Bolivariana de Venezuela

36 También hay asuntos relacionados con Paraguay que parecen tener final feliz, se trata en primer lugar de unas comunidades indígenas enxet-lamenxay y kayley-phapopyet -Riachito. Vid. Comunidades Indígenas Enxet Lamenxay y KaleyphapopyetRiachito v. Paraguay, Caso 11.713, Informe No. 90/99, OEA/Ser.L/V/II.106 Doc. 3 rev. at 350 (1999). A partir de 1885 el Gobierno paraguayo comenzó a vender todas las tierras del Chaco a no-indígenas y en 1950 todo el territorio enxet había sido ocupado por los nuevos propietarios. Estas comunidades indígenas han logrado una solución amistosa consistente en que el Estado paraguayo se comprometió (mediante un acuerdo promovido, por la Comisión Interamericana de Derechos Humanos, celebrado con las Comunidades interesadas) a adquirir de nuevo las tierras en cuestión (21.884'44 Has. en el Chaco paraguayo), con el objeto de transferírselas a las comunidades indígenas interesadas y registrarlas bajo su propiedad colectiva. (donde se cede territorio para construir comunidades indígenas socialistas ${ }^{37}$ ), también hay datos alentadores en este sentido de Rusia ${ }^{38}$. Sin embargo, el establecimiento y la titulación de las tierras indígenas, cuando se produce, lejos de ser el final de los problemas, resulta el inicio de otros. Estos territorios poseen recursos naturales no explotados (cuya propiedad defienden los pueblos indígenas y rechazan los Estados), por tanto, no puede extrañarnos que personas

37 Sobre los pueblos indígenas en Venezuela, vid. S. Torrecuadrada, Los derechos de los pueblos indígenas en Venezuela, Madrid, Biblioteca Nueva, 2009.

38 Otro caso ilusionante en relación con los derechos sobre las tierras es el de los inuit canadienses, que han conseguido negociar con las autoridades nacionales un régimen de autonomía política (Nunavut, más de 2 millones de $\mathrm{km} 2$, con menos de 30.000 habitantes, al noroeste de Canadá) en virtud del acuerdo celebrado en 1994. En otros lugares, se alcanza la demarcación de sus tierras ancestrales o cuando menos la proclamación constitucional o legal de esta operación, como en el caso de Venezuela (aunque sea para constituir comunidades indígenas socialistas), Brasil o el mismo Paraguay. Sin embargo, en muchos lugares, estos reconocimientos constitucionales son más formales que reales debido a que la falta de desarrollo normativo o reglamentario impide el goce efectivo de los derechos objeto de reconocimiento; o, de existir, ese desarrollo normativo contiene mecanismos ineficaces para acceder a la titulación de las tierras. El caso de Rusia también es ilustrativo, dado que el «Código de Tierras adoptado en 2001 permite la apropiación privada de la tierra, pero establece mecanismos tan onerosos para acceder a la propiedad que la mayoría de las comunidades indígenas han quedado excluidas del proceso». 
ávidas de riqueza contemplen la facilidad de conseguir pingües beneficios con poco esfuerzo, adentrándose en las tierras indígenas para explotar sus riquezas. En ocasiones, estas intromisiones en territorio indígena tienen consecuencias terribles para los miembros del grupo. Es el caso de Brasil, donde el afán de los garimpeiros produjo la invasión de las tierras yanomamis en busca de oro, propósito por el que no dudaron en matar a quienes se oponían a la ocupación. Estos comportamientos desplegaron efectos indirectos, ya que el contacto produjo el contagio de enfermedades a las que no eran inmunes los indígenas, resultando mortales para ellos $^{39}$. En la actualidad es un grupo en peligro de extinción ${ }^{40}$.

39 El origen de esta situación se encuentra en la construcción de la autopista transamazónica que atraviesa los territorios donde viven los yanomamis (teóricamente inalienables precisamente por la condición de sus habitantes). Tierras sobre las que, de acuerdo con la Constitución brasileña, tienen derecho a la posesión permanente y al usufructo exclusivo de las riquezas naturales y beneficios que en ellas puedan obtenerse. Durante el período de construcción de la autopista se descubrieron ricos minerales en las tierras yanomamis, motivo por el cual colonos no indígenas ávidos de riqueza rápida las invadieron violentamente para aprovecharse de sus recursos. Vid. Resolución 12/85 de la Comisión en el caso $\mathrm{n}^{\circ} 7615$, de 5 de marzo de 1985 , en la que la Comisión Interamericana formula recomendaciones a Brasil para que intente reparar la situación creada.

Los yanomamis venezolanos también han sido víctimas de la civilización ya que, de acuerdo con Tierney P. (2000), Darkness in el Dorado. How Scientists and Journa-
Desafortunadamente, lejos de ser casos aislados, las incursiones en territorios indígenas para beneficiarse de los recursos existentes muestran múltiples manifestaciones, piénsese que en los territorios indígenas se han encontrado oro (en Guatemala), níquel (en Nueva Caledonia), gas (en la amazonía peruana), petróleo (Chad y Camerún) para cuya extracción los interesados no dudan en desplazar violentamente a los pueblos indígenas. Cuando no se les expulsa de sus territorios ancestrales, las técnicas aplicadas a la extracción de los recursos son muy contaminantes, lo que unido a la utilización de sus territorios como depósito de residuos tóxicos, ha producido el aumento importante de la incidencia de graves enfermedades, como el cáncer, en las comunidades afectadas. Esto, añadido a la escasez de recursos y a las dificultades para acceder al sistema sanitario nacional, produce un resultado fácilmente imaginable. Lo anterior evidencia que la autorización para la exploración y explotación de los recursos de los territorios indígenas ha de relacionarse necesariamente con su propiedad. Lo contrario imposibilita el pacífico disfrute de sus derechos en este ámbito material, amenazándose el más básico de los derechos humanos: la subsistencia.

El excesivo alcance del que se dota al consentimiento libre e informado

lists Devastated the Amazon, Nueva York, W.W. Norton y Co han sido utilizados como cobayos por un grupo de científicos estadounidenses. 
de los pueblos indígenas ${ }^{41}$ es otro de los motivos de oposición estatal a la Declaración (alegado por Canadá, por ejemplo), que establece la necesidad de obtener el consentimiento indígena para adoptar o ejecutar medidas que potencialmente afectarían a los pueblos considerados ${ }^{42}$. Regulación con la que se estaría discriminando al resto de la sociedad que puede manifestar su opinión exclusivamente por conducto de los cauces institucionalmente establecidos para ello, aunque la decisión les afecte en forma directa; careciendo del derecho de veto (ausencia de consentimiento) del que se dota a sus compatriotas indígenas cuando los textos normativos en cuestión les afecten directa o en indirectamente. En todo caso, la interpretación literal de la Declaración en este punto no resulta acorde con la defendida por el Comité de Expertos de la OIT, que considera este consentimiento un mecanismo de expresión idóneo para los pueblos indígenas y tribales, con el objeto de influir en el proceso de adopción de decisiones estatales, pero sin implicar un derecho de veto ${ }^{43}$.

41 Es el artículo 18 de la Declaración: «Los pueblos indígenas tienen derecho a participar en la adopción de decisiones en las cuestiones que afecten a sus derechos, por conducto de representantes elegidos por ellos de conformidad con sus propios procedimientos, así como a mantener y desarrollar sus propias instituciones de adopción de decisiones».

42 Vid. explicación de voto del representante canadiense en A/61/PV.107.

43 Vid. en http://www.ilo.org.
La primacía del derecho consuetudinario sobre el derecho nacional es un aspecto que preocupa a Australia ${ }^{44}$. La Declaración se refiere a la Ley (entendiendo por ella la nacional) exclusivamente en su último artículo (art. 46.2) para afirmar que «El ejercicio de los derechos establecidos en la presente Declaración estará sujeto a las limitaciones determinadas por la ley y con arreglo a las obligaciones internacionales en materia de derechos humanos». Del texto transcrito puede deducirse que el límite legal existe, aunque queda por determinar si todos los ámbitos contenidos en la Declaración pertenecen a la categoría de derechos humanos. De ser así nos encontraríamos ante un límite más ficticio que real aunque protegido por el efecto del texto en el que se incorpora la Declaración (una resolución de la asamblea general), insuficiente por sí misma para crear obligaciones jurídicamente exigibles. La interpretación del precepto en sus justos términos no plantea tales problemas, debiendo considerar como límite de la Ley nacional el núcleo duro de los derechos humanos consolidados como tales desde la perspectiva internacional.

En la opinión de algunos Estados, la excesiva amplitud de los derechos que se conceden a los pueblos indígenas (especialmente la exclusividad de la que se dota a la propiedad intelectual y de las tierras), provoca el desconocimiento de los derechos de terceros a pesar

${ }_{44}$ Vid. explicación de voto del representante australiano en A/61/PV.107. 
de lo que establezcan las legislaciones nacionales (según Canadá o Nueva Zelandia). Cierto es que en el artículo 46, la Declaración se refiere tímidamente a los derechos de los terceros ${ }^{45}$, aunque su redacción parece indicar la prevalencia de los derechos indígenas sobre aquellos de los demás, que solo ceden frente a los derechos humanos de terceros exclusivamente para satisfacer las necesidades básicas de la sociedad democrática. Aunque el Estado goce de discrecionalidad para calificar cuando nos encontramos en presencia de una situación de este tipo, es evidente que la redacción despierta sospechas entre aquellos que cuentan con población indígena puesto que, salvo en circunstancias excepcionalísimas, parecen ignorarse los derechos de terceros.

Por último, en opinión del representante canadiense, se caracteriza por ser la relación entre las obligaciones y los derechos de los pueblos indígenas y, convengamos que, si bien son muchos los derechos de titularidad indígenas y que estos son titulares de un amplio abanico de derechos añadidos a los del resto (de conformidad con la Declara-

45 El artículo 46 apunta que las limitaciones derivadas de la ley y del derecho internacional en materia de derechos humanos «no serán discriminatorias y serán solo las estrictamente necesarias para garantizar el reconocimiento y respeto debidos a los derechos y las libertades de los demás y para satisfacer las justas y más apremiantes necesidades de una sociedad democrática», lo que es no decir nada o decir demasiado. ción), y las obligaciones a ellos dirigidas son muy escasas.

\section{B. Los inconvenientes que pueden derivar del resultado de la votación para la consolidación de la Declaración como derecho internacional general}

La Declaración de Naciones Unidas sobre los Derechos de los pueblos indígenas es un anexo a una resolución de la Asamblea General y, en consecuencia, cuenta con los efectos derivados del instrumento en el que se incorpora, carente de fuerza obligatoria. A pesar de ello, se realiza con la esperanza de que siguiera el camino de la Declaración Universal de Derechos Humanos que, con el transcurso del tiempo, no solo se consolidó como derecho internacional general oponible a todos los Estados sino, en algunos aspectos, con carácter imperativo. Sin embargo, el punto de partida de un texto y otro difieren en un aspecto esencial: en 1948 la Declaración se adoptó sin votos en contra y con ocho abstenciones ${ }^{46}$; por el contrario, el texto de 2007 recibió 143 votos a favor (34 Estados estuvieron ausentes en la sesión de votación), 11 abstenciones y 4 votos en contra ${ }^{47}$.

46 Sudáfrica, Arabia Saudita, Bielorrusia, Checoslovaquia, Polonia, Ucrania, la Unión de República Social Soviética y Yugoslavia.

47 Las abstenciones de Azerbaijan, Bangladesh, Bhutan, Burundi, Colombia, Georgia, Kenya, Nigeria, Federación de Rusia, 
Podemos pensar que si la Declaración se consolida como derecho internacional general, el comportamiento de los Estados Miembros de las Naciones Unidas en la votación puede analizarse como indicio de la oponibilidad de los derechos contenidos en ella. De modo que, quienes votaron en contra han salvaguardado su posible posición como objetor persistente, siempre que su comportamiento posterior lo avale.

Ciertamente, con ánimo de evitar posibles oposiciones podía haberse elaborado un texto menos ambicioso pero que permitiera la adopción de la Declaración por consenso, de modo de facilitar la producción de efectos generadores de normas consuetudinarias. Creo que es mejor obtener un consenso de mínimos que permita su consolidación a los efectos perseguidos, que aprobar un texto ambicioso exclusivamente con valor recomendatorio. Sin embargo, lo que se buscaba era el compromiso estatal en el respeto de los derechos allí incorporados. Si Estados que habiendo votado a favor del texto (tomemos como ejemplo a los latinoamericanos salvo Colombia, que se abstuvo), vulneran directa o indirectamente los derechos indígenas más básicos (léase el derecho a la vida), rebajando el estándar mínimo de protección, no conseguiría un nivel de compromiso superior en la garantía de esos derechos, reiteradamente vulnerados.

Samoa y Ucrania y los votos en contra de Australia, Canadá, Estados Unidos y Nueva Zelandia.
Pero ¿qué necesitamos para que la Declaración se consolide como lo hizo la Declaración Universal de 1948? El Prof. Remiro indica que hubo factores originarios y sobrevenidos al texto que «determinan y confirman» su valor jurídico $^{48}$. Entre los primeros (originarios) la Declaración de 2007 reúne dos de los tres presentes en su predecesora: en primer lugar, se trata del "contenido vocacionalmente normativo", al desprenderse de su redacción la «intención de enunciar principios jurídicos»; en segundo término, se incorpora en una resolución-declaración de la Asamblea General instrumento con el que este órgano ha enunciado principios vocacionalmente jurídicos con lo que se crea una «fuerte expectativa de que serán cumplidas por los miembros de la comunidad internacional» ${ }^{49}$ que, de cumplirse, se consolidarán como normas consuetudinarias generales. El tercero de los factores a considerar aleja ambas Declaraciones, al tratarse del apoyo recibido al tiempo de su adopción, aunque ninguna de ellas fue aprobada por unanimidad ni por consenso y el apoyo recibido por ambas superó los dos tercios de los miembros de la Organización $^{50}$. En 1948 se produjeron

48 Vid. Remiro Brotóns A. y otros, Derecho Internacional, Madrid, Tirant Loblanch, 2007, pp. 1426-27.

49 Id.

so El art. 18.2 de la Carta de Naciones Unidas establece que «Las decisiones de la Asamblea General en cuestiones importantes se tomarán por el voto de una mayoría de dos tercios de los miembros presentes y votantes». La mayoría de 2/3 se obtuvo 
algunas abstenciones (un $13.8 \%$ de los 58 miembros con los que contaba la Organización); en 2007 cuatro Estados votaron contra la Declaración, lo que supone la importante diferencia entre ambos textos en lo que a los factores originarios se refiere ${ }^{51}$.

La segunda categoría de elementos a observar son los sobrevenidos, considerándose tales los comportamientos estatales o institucionales posteriores a la adopción del texto en cuestión. Buscando comprobar la presencia de la práctica estatal, en el caso de la Declaración de 1948, se consideran en este punto las referencias a ellas en otros tratados internacionales o en las Constituciones estatales o la «invocación de sus disposiciones en orden a legitimar la actuación internacional sin base convencional que tiene lugar ante la violación grave y sistemática y de los derechos humanos» ${ }^{52}$. Evidentemente,

sobradamente tanto si consideramos lo establecido en el precepto recién transcrito (2/3 de miembros presentes y votantes) como si la totalidad es la del número de Estados miembros.

51 El resultado de la votación de 2007 en términos porcentuales muestra un apoyo porcentual inferior, logrando un 74,48\% de los 192 Estados miembros. Igualmente desciende el índice porcentual de la abstención, que se ubica en un $5.73 \%$, mientras aparecen las ausencias en la sesión de votación (un $177 \%$ ) y los votos contrarios, pese a que únicamente suponen un $2.09 \%$ del total de los miembros.

52 Vid. la Sentencia de la Corte Interamericana de Derechos Humanos, de 27 de noviembre de 2007, en el asunto del pueblo Saramaka contra Suriname, nota 134 , en p. 44. es muy temprano para comparar lo ocurrido en relación con la Declaración sobre los derechos de los pueblos indígenas. En adelante habrá que observar a los órganos nacionales e internacionales encargados de la aplicación del derecho para comprobar la utilización que hacen de este texto. De momento, los inicios han sido buenos: dos meses después de su adopción, la Corte Interamericana de Derechos Humanos la utilizó junto con el Convenio 169 de la OIT en relación con la necesidad de consulta previa e informada de la que son titulares los pueblos indígenas ${ }^{53}$.

A pesar de la voluntad de establecer normas jurídicas o de elevar el nivel de protección del que han de ser titulares los pueblos indígenas, desde una perspectiva de análisis jurídico-internacional, el efecto de la Declaración no puede ser diferente del apuntado. Así, por mucho que compartamos el deseo de que el contenido de la Declaración sea jurídicamente exigible a los Estados miembros de la Organización, no podemos considerar acertado el contenido del Comentario del Foro permanente sobre cuestiones indígenas en este punto $^{54}$. En este Documento se interpreta

53 Vid. «Artículo 42 de la Declaración de las Naciones Unidas sobre los Derechos de los pueblos indígenas», publicado en el Informe sobre el octavo periodo de sesiones, del 18 al 29 de mayo de 2009, Doc. E/C. 19/2009/14.

54 El artículo 42 de la Declaración indica que «Las Naciones Unidas, sus órganos, incluido el Foro Permanente para las Cuestiones Indígenas, y los organismos especializados, incluso a nivel local, así como 
el artículo 42 de la Declaración ${ }^{55}$, que encomienda a las Naciones Unidas la tarea de promover el respeto de este texto, entendiendo que es jurídicamente vinculante para todos los Estados con independencia del sentido de su voto, de lograr la materialización de los deseos de sus autores, en un ejercicio de interpretación imposible. Quizá el transcurso del tiempo nos proporcione los efectos jurídicos allí establecidos, restándonos un largo camino por recorrer para lograrlo.

Desde la perspectiva del derecho internacional, la opinión del Foro Permanente en algunos aspectos es fácilmente rebatible, aunque nos duela, en primer lugar porque la Asamblea General es un órgano carente de competencias legislativas. En consecuencia, los instrumentos que se aprueban en su seno solo pueden adquirir efectos jurídicamente obligatorios de cumplirse los elementos antes señalados. Puede establecerse un paralelismo entre el procedimiento empleado para la negociación de la Declaración y la celebración de los Tratados internacionales. De ser así, la

los Estados, promoverán el respeto y la plena aplicación de las disposiciones de la presente Declaración y velarán por su eficacia".

55 El artículo 42 de la Declaración indica que «Las Naciones Unidas, sus órganos, incluido el Foro Permanente para las Cuestiones Indígenas, y los organismos especializados, incluso a nivel local, así como los Estados, promoverán el respeto y la plena aplicación de las disposiciones de la presente Declaración y velarán por su eficacia». votación sería semejante a la adopción del texto del tratado, del que únicamente deriva la obligación de no frustrar su objeto y fin. La opinibilidad del tratado es consecuencia de la manifestación del consentimiento particular de los Estados, una vez cumplidos los cauces constitucionalmente establecidos para ello y la posterior entrada en vigor del texto convencional.

En otro orden de consideraciones, no cabe interpretar el comportamiento de un representante estatal ante la adopción de una resolución de la Asamblea General como manifestación del consentimiento en obligarse por ella, del mismo modo, no es posible derivar este efecto del voto en la adopción de un tratado internacional, entre otras razones porque el procedimiento normativo establecido con carácter general así lo establece, sin que existan materias en las que se aplica de modo diferente. Es cierto que hay obligaciones que se imponen a los Estados con independencia de su manifestación del consentimiento en obligarse por ellas, son las normas imperativas que en la medida en que estén presentes en la Declaración se benefician de esta naturaleza. Sin embargo, no es posible considerar que la totalidad de su contenido se vea alcanzado como ius cogens, dado que el complicado procedimiento de identificación de este tipo particular de normas no puede considerarse cubierto con la mera adopción de la Declaración.

Tampoco puede afirmarse que el contenido de la Declaración sea derecho consuetudinario internacional; 
la votación en la Asamblea General puede ser el elemento espiritual de la costumbre (opinio iuris) pero para que exista una norma consuetudinaria se precisa junto a este el elemento material: la práctica estatal. A pesar de que hay elementos que nos permiten afirmar la suma de ambos elementos en algunos derechos, no es posible, al menos en diciembre de 2009, afirmar que la totalidad de la Declaración expresa derecho consuetudinario internacional, y muchísimo menos que sea exigible a todos los Estados, con independencia del sentido de su voto. No podemos olvidar la figura del objetor persistente que es, a la costumbre internacional, lo que a un tratado la falta de manifestación del consentimiento en obligarse. Hemos de observar el comportamiento de los cuatro Estados que votaron en contra para intentar identificar posibles fisuras que nos permitan afirmar la pérdida de la condición de objetor persistente que avanzaron con su voto en la aprobación de la Declaración, para evitar su oponibilidad si se consolida como norma consuetudinaria.

También alude el mismo documento del Foro Permanente al artículo 38 del Estatuto de la Corte Internacional de Justicia, en el que encontramos lo más parecido a una relación de las fuentes del derecho internacional, para considerar el encaje del contenido de la Declaración en la categoría de principios generales del derecho. Sin embargo, el artículo 38 se refiere a los instrumentos de derecho internacional que aplicará la Corte a la solución de una controversia cuyo conocimiento se le haya sometido. Por lo demás, sabido es que los principios generales del art. 38 del Estatuto no son los principios estructurales sobre los que se edifica el ordenamiento internacional, sino los principios de naturaleza procesal importados de los ordenamientos internos con los que se intentan colmar las lagunas existentes en el derecho internacional.

\section{REFLEXIONES FINALES}

Desde septiembre de 2007 contamos con un texto en el que se reconocen los derechos de los pueblos indígenas, sin que con ello hayan terminado los problemas a que han de enfrentarse estos grupos humanos. Observemos el caso de los indígenas colombianos, ecuatorianos o peruanos, por citar solo algunos de los ubicados en América Latina. Bien al contrario, en muchas ocasiones, el reconocimiento formal de derechos es generador de nuevos problemas en la medida en que la materialización del objeto reconocido no se produce, o se produce sin garantías, lo que impide el ejercicio del derecho del que se es titular.

En el ámbito de los derechos de los pueblos indígenas contamos, en relación con los derechos que más les interesan (de naturaleza colectiva y de forma particular los relacionados con el elemento territorial), con textos que, como la Declaración de las Naciones Unidas, carecen de efectos jurídicamente vinculantes o con tratados. Los 
Convenios de la OIT que, si bien los poseen, carecen sistemas específicos de control de cumplimiento.

Los Estados, en muchas ocasiones no adaptan su legislación para cumplir con las obligaciones internacionalmente contraídas o, de hacerlo, no adoptan la normativa reglamentaria necesaria para ello, o escudan su incumplimiento en la falta de recursos financieros u otros. Es el caso de Colombia que se abstuvo en la votación final a pesar de haber reafirmado en la Asamblea General su compromiso con los derechos de las comunidades indígenas. La abstención colombiana estaba motivada por el imprescindible control efectivo de todo el territorio colombiano por parte del ejército, también en los territorios indígenas para su protección. Tristemente las comunidades indígenas se encuentran en el centro de un triángulo de horror y terror formado por la guerrilla, los paramilitares y el ejército. El caso colombiano ilustra la denominada brecha entre las normas internacionales en materia de derechos humanos indígenas y la legislación interna que, por razones de seguridad nacional en este caso, se ve imposibilitada de asumir el compromiso derivado de las disposiciones de la Declaración. No es este el único aspecto incumplido (y no solo por Colombia), también el de la educación bilingüe, para cuya implantación con éxito se precisa de financiación de la que, en unos casos, no se dispone y, en otros, se pierde entre corruptelas internas, ampliándose cada vez más la brecha en la implementación.
En otras ocasiones, lo que ocurre es que el reconocimiento de los derechos indígenas no se compatibiliza con otros cuerpos legislativos, como la ley de minas, por ejemplo. Por una parte se reconoce la propiedad indígena sobre sus respectivos territorios y sobre los recursos que se encuentren en ellos, sin adecuarse los cuerpos legislativos internos a aquellos, es decir, no se excluyen los territorios indígenas de la aplicación de otras normas nacionales que regulan con carácter general la explotación y exploración de los recursos. En los supuestos en que ese reconocimiento sea un acto unilateral internacional o derive de un tratado internacional, resulta jurídicamente exigible la adaptación a él del ordenamiento interno, por lo que el mantenimiento de la legislación en contrario es un ilícito internacional del que deriva responsabilidad internacional.

Otros Estados (especialmente asiáticos y africanos) niegan la existencia en sus territorios de pueblos indígenas. Con este punto de partida, la consecuencia de la ausencia de población indígena es la inexistencia en sus territorios de titulares de los derechos reconocidos, desapareciendo el incumplimiento.

Con todos los problemas que podamos identificar, no podemos dejar de afirmar que uno de los grandes éxitos de los pueblos indígenas desde el comienzo de los años noventa, cuando se declara el Primer Decenio Internacional de los pueblos indígenas, anterior por tanto a la Declaración y, que probablemente se encuentra en el origen de su adopción, 
ha sido la pérdida de su invisibilidad. En estos años han adquirido visibilidad interna e internacional. Interna porque en las constituciones estatales han aparecido también los pueblos indígenas aunque en ocasiones resulten reconocimientos más formales que reales; Internacional, al haberse avanzado en la participación indígena en los foros internacionales. Se han dado cuenta de las posibilidades que les ofrece la situación internacional actual, permitiéndoles un horizonte diferente a la mera asimilación. Así, el conocimiento de la existencia de otros pueblos indígenas ha proporcionado su organización y movilización social, con el objeto de hacer escuchar sus demandas.

Es verdad que los pueblos indígenas han sido los grandes ausentes en la construcción de las sociedades estatales en cuyo territorio habitan. Pero también lo es que la vuelta a la situación anterior a la conquista y colonización de sus territorios es imposible. Por tanto, la solución que nos queda es la de intentar negociar una solución adecuada para todas las partes interesadas, cuyas bases han de negociarse desde la Declaración de 2007. Ahora nos queda solo esperar a que la práctica estatal posterior a su adopción permita la consolidación definitiva y real de su contenido como norma general de derecho internacional.

\section{REFERENCIAS BIBLIOGRAFICAS}

Aguilar Cavallo, G. (2005), "El título indígena y su aplicabilidad en el Derecho chileno", en Ius et Praxis, vol. 11, n. 1, pp. 269-295.

Aparicio Wilhelmi, M. (2006), «Los pueblos indígenas y sus demandas, punto de partida del reconocimiento constitucional de los derechos indígenas» en M. Carrasco Durán y otros (coords.), Derecho constitucional para el siglo XXI: actas del VIII Congreso Iberoamericano de Derecho Constitucional, vol. 2, Sevilla, Aranzadi, pp. 4939-4948.

BerRaONDo, M. (2004), «Derecho humano al medio ambiente y pueblos indígenas. Dos derechos con un mismo fin", en J. D. Oliva Martínez y F. M. Mariño Menéndez (coords.) Avances en la protección de los derechos de los pueblos indígenas, Madrid, Dyckinson, pp. 73-88.

—_ (2005), «Los retos de la agenda indígena ante el nuevo decenio", en Papeles de cuestiones internacionales, n. 90, pp. 109-116.

- (2005), «Pueblos indígenas y recursos naturales bajo el sistema interamericano de derechos humanos: entre la privatización y el ejercicio de los derechos humanos», M. Aparicio Wilhelmi (coord.), en Caminos hacia el reconocimiento: pueblos indigenas, derechos y pluralismo, Universitat de Girona, pp. 141-180.

- (2006), «Tierras y territorios como elementos sustantivos del Derecho Humano al medio ambiente», en M. BERRAONDO (coord.) Pueblos indígenas y derechos humanos, Universidad de Bilbao, Deusto, pp. 469-488.

Dávalos, P. (2002), «Movimiento indígena ecuatoriano: Construcción política y epistémica", en D. Mato (coord.), Estudios y Otras Prácticas Intelectuales Latinoamericanas en Cultura y Poder, Consejo Latinoamericano de Ciencias Sociales (CLACSO) y CEAP, FACES, Caracas, Universidad Central de Venezuela, pp: 21-46. 
Gómez IsA, F. (ed.) (2003), El caso Awas Tingni contra Nicaragua. Nuevos horizontes para los derechos humanos de los pueblos indígenas, Bilbao.

Grupta, A. (1998), Postcolonial Developments. Agricultur in the making of modern India, Dirham, Duke University Press.

KIRK, M. "Should the United Status ratify the American Convention on Human Rights?", Revista del Instituto Interamericano de Derechos Humanos, n. 14, pp. $65-89$.

Joy Hassol, S. (2004), Impacts of a Warming Arctic, Cambridge, Cambridge University Press.

Lázaro Calvo, T. (2005), Derecho Internacional del Medio Ambiente, Barcelona, Atelier.

Mariño, F.M. y Oliva, J.D. (eds.) (2004), Avances en la protección de los derechos de los pueblos indígenas, Madrid, Dyckinson.

Martínez de Bringas, A. (2003), Los pueblos indígenas y el discurso de los derechos, Universidad de Bilbao, Deusto.

Triggs, G. (1998), «Peoples'Rights and Individual Rights", en J. Crawford, The Rights of Peoples, Oxford University Press.

Torrecuadrada García-Lozano, S. (2001), Los pueblos indígenas en el orden internacional, Madrid, Dyckinson.

- (2004), «La situación jurídica internacional de los pueblos indígenas», en Cursos de derechos humanos de DonostiaSan Sebastián, vol. 5, pp. 335-363

__. (2006), «La consideración internacional de las poblaciones indígenas en los últimos años: evolución y asignaturas pendientes", Themis, n. 52, pp. 269-285.

--_---. (2008), "Cambio climático y pueblos indígenas», en A. Remiro Brotóns y R.M. Fernández Egea (eds.), El Protocolo de Kioto: Dificultades de su aplicación en el Derecho Internacional y Comunitario, Madrid, Fundación BBVA. pp. 291-316.

- (2009), Los derechos de los pueblos indígenas en Venezuela, Madrid, Biblioteca Nueva.
UlloA, A. (2001), «El nativo ecológico: Movimientos indígenas y medio ambiente en Colombia", en Archiva, M. Y M. Pardo (eds.), Movimientos sociales, estado y democracia en Colombia, Bogotá, ICANHCES-Universidad Nacional.

- (2004), La construcción del nativo ecológico: complejidades, paradojas y dilemas de la relación entre movimientos indígenas y ambientalismo en Colombia, Bogotá, Flacso.

\section{Documentación:}

Informes de Naciones Unidas:

Informe del Relator Especial de Naciones Unidas, Sr. Martínez Cobo, sobre la discriminación en perjuicio de las poblaciones indígenas y recomendara la adopción de medidas nacionales para eliminarlas. Vid. en (UN Doc. E/CN.4/Sub.2/1986/7/ Add.4).

Informe del Relator Especial de Naciones Unidas sobre la situación de los derechos humanos y libertades fundamentales de los pueblos indígenas, Rodolfo Stavenhaguen, presentado de conformidad con la resolución 2001/65 de la Comisión el 21 de enero de 2003, NU, Doc. E/CN.4/2003/90,

Informe del Secretario General sobre el examen preliminar realizado por el Coordinador del Decenio Internacional de las Poblaciones Indígenas del Mundo de las actividades del sistema de las Naciones Unidas en relación con el Decenio, de 25 de junio de 2004, en Doc. E/2004/82.

Informe del Tercer Período de Sesiones del Foro Permanente para las cuestiones indígenas, reunido entre los días 10 y 21 de mayo de 2004, en Doc. E/C19/2004/23.

Informe del Relator Especial de Naciones Unidas sobre la situación de los Derechos Humanos y las libertades fundamentales de los indígenas, Rodolfo Stavenhagen al Consejo de derechos Humanos, Doc. A/ HRC/4/32, de 27 de febrero de 2007. 
Resoluciones de la Asamblea General de las Naciones Unidas (AGNU):

Resolución 48/163, adoptada por la AGNU el 21 de diciembre de 1993, sobre el Primer Decenio internacional de las poblaciones indígenas del mundo.

Resolución 50/157, adoptada por la AGNU el 21 de diciembre de 1995, que contiene el Programa amplio a desarrollar durante el Primer Decenio internacional de las poblaciones indígenas del mundo.
Resolución 59/174, adoptada por la AGNU el 20 de diciembre de 2004, sobre el segundo decenio internacional de las poblaciones indígenas del mundo.

Resolución 61/295 de la AGNU, adoptada por la AGNU el 10 de diciembre de 2007, que contiene la Declaración de las Naciones Unidas sobre los Derechos de los pueblos indígenas. 